\title{
CoVR: A Large-Scale Force-Feedback Robotic Interface for Non-Deterministic Scenarios in VR
}

Elodie Bouzbib ${ }^{1,2}$

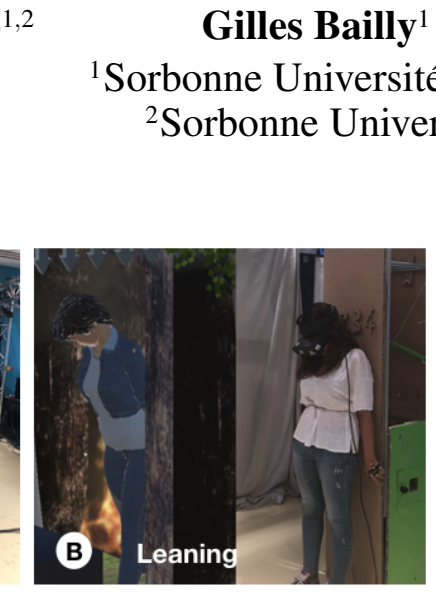

Sinan Haliyo ${ }^{1}$

Pascal Frey ${ }^{2}$

, CNRS, ISIR. Paris, France

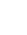

, ISCD. Paris, France
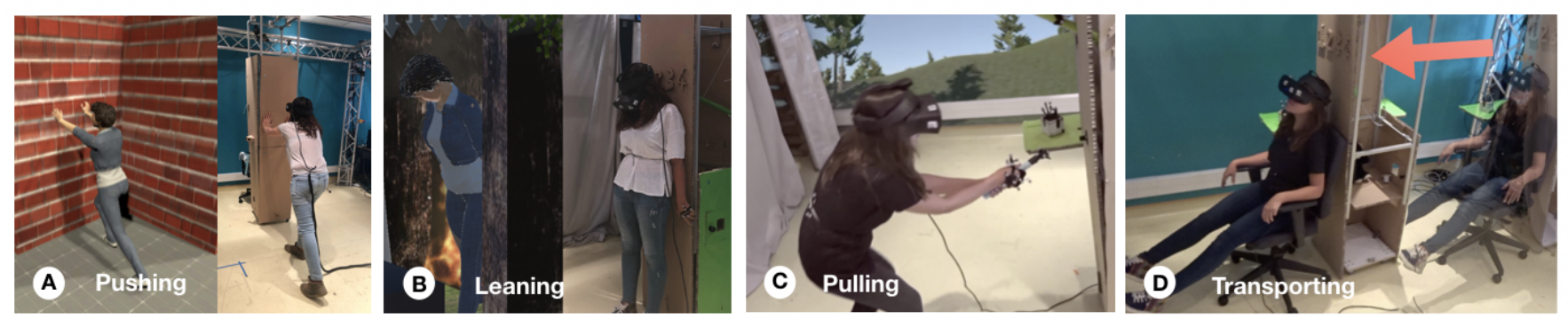

Figure 1. CoVR is a physical column mounted on a 2D Cartesian ceiling robot to provide strong kinesthetic feedback (> 100N) in a room-scale VR arena. The column panels are interchangeable and its movements can safely reach any location in the VR arena thanks to XY displacements and trajectory generations avoiding collisions with the user. When CoVR is static, it can resist to body-scaled users' actions, such as (A) users pushing on a static tangible rigid wall with a high force or (B) leaning on it; When CoVR is dynamic, it can act on users. (C) CoVR can pull the users to provide large force-feedback or even (D) transport the users.

\begin{abstract}
We present CoVR, a novel robotic interface providing strong kinesthetic feedback $(100 \mathrm{~N})$ in a room-scale VR arena. It consists of a physical column mounted on a 2D Cartesian ceiling robot (XY displacements) with the capacity of (1) resisting to body-scaled users' actions such as pushing or leaning; (2) acting on the users by pulling or transporting them as well as (3) carrying multiple potentially heavy objects (up to $80 \mathrm{~kg}$ ) that users can freely manipulate or make interact with each other. We describe its implementation and define a trajectory generation algorithm based on a novel user intention model to support non-deterministic scenarios, where the users are free to interact with any virtual object of interest with no regards to the scenarios' progress. A technical evaluation and a user study demonstrate the feasibility and usability of CoVR, as well as the relevance of whole-body interactions involving strong forces, such as being pulled through or transported.
\end{abstract}

\section{Author Keywords}

Virtual Reality; Haptics; Kinesthetic Feedback; Actuated device; Tangible User Interface; Encountered-type Haptic Devices; Robotic Graphics

Permission to make digital or hard copies of all or part of this work for personal or classroom use is granted without fee provided that copies are not made or distributed for profit or commercial advantage and that copies bear this notice and the full citation on the first page. Copyrights for components of this work owned by others than the author(s) must be honored. Abstracting with credit is permitted. To copy otherwise, or republish, to post on servers or to redistribute to lists, requires prior specific permission and/or a fee. Request permissions from permissions@acm.org.

UIST '20, October 20-23, 2020, Virtual Event, USA

(C) 2020 Association for Computing Machinery.

ACM ISBN 978-1-4503-7514-6/20/10 ...\$15.00.

http://dx.doi.org/10.1145/3379337.3415891

\section{INTRODUCTION}

While visual and auditory displays in Virtual Reality (VR) have reached a level where the produced stimuli are quite convincing, haptic technology is still poor compared to the rich ways humans can interact with their environment. Multiple directions have been envisioned to enhance the users' haptic experiences in VR, through hand-held controllers or wearables simulating the environment $[22,11]$, or through the direct manipulation of passive props [37, 14].

In these regards, McNeely introduced Robotic Graphics and more specifically Robotic Shape Displays (RSD) [45] in 1993 as a concept for providing force-feedback in VR. It explores the use of a robotic interface in the VR arena to provide haptic experiences, while the user remains unencumbered (no wearables, no controllers) [67]. It aims to stay out of reach when no interaction is required, and to displace passive props to dynamically overlay virtual ones otherwise.

Multiple expanding fields such as Gaming, Training or Simulation could benefit from this concept. Several approaches have already been conducted to instantiate it, from human actuators [19] to drones [5]. However, they often suffer from several technical trade-offs (e.g. cost, real workspace, embedded mass, speed, accuracy) that grow into interaction challenges (e.g. locomotion whole body interaction, force-feedback, free manipulation of multiple props).

In this paper, we propose $\mathrm{CoVR}^{1}$, a novel Robotic Shape Display providing whole-body interactions and strong force

${ }^{1}$ CoVR, pronounced "Cover", stands for a Column in VR which physically covers for virtual objects 
feedback in room-scale arenas. We detail our approach from three complementary perspectives:

From a mechanical perspective, CoVR is a 2D Cartesian ceiling robot, carrying a column. With only two single degrees of freedom (XY displacements), CoVR is a large-scale grounded robot which exhibits notable mechanical capabilities: high lateral and vertical force feedback and perceived stiffness (over $100 \mathrm{~N}$ ) and load capabilities (over $80 \mathrm{~kg}$ ). It hence can transport a variety of potentially heavy props and objects to stand in for their virtual counterparts. We demonstrate that our implementation is fast (over $1.0 \mathrm{~m} / \mathrm{s}$ ) and accurate (under $2 \mathrm{~cm}$ in a $30 \mathrm{~m}^{3}$ arena).

From a software perspective, while it is easy to control Cartesian robots as they only rely on XY displacements, defining the robot target positions can be challenging. The system has to predict where the next interaction will occur, while avoiding the user for unexpected collisions. We thus elaborate a user intention model to make CoVR always available prior to the users' interactions, even in non-deterministic scenarios, i.e. where users are free to interact with any virtual object of interest with no regards to the scenarios' progress. Given the position and orientation of the user as well as the objects of interest's, the model estimates the best positions for the robot and its optimal trajectories to reach an object of interest while respecting safety constraints (e.g. safe-zones around the user).

A technical evaluation demonstrates that (1) user intentions can be captured using available data from a single HMD with no additional apparatus in a room-scale VR arena and that (2) our system successfully reaches targets prior to interactions in most of non-deterministic scenarios with randomly distributed targets and distractors.

From the interaction perspective, CoVR offers (1) body-scaled interactions such as leaning or pushing involving strong forces with a static column (Figure 1 - A, B), or (2) dynamic interactions such as being pulled through by large traction forces (Figure 1 - C). CoVR can displace over $80 \mathrm{~kg}$ of embedded mass, which enables (3) interactions with potentially heavy objects that users can freely manipulate but also (4) transport of the users themselves (Figure 1 - D).

We report on a user study demonstrating (1) the robustness of both CoVR mechanical and software implementations, (2) the benefits of a robotic interface providing body-scale interactions and strong forces and (3) in particular "being transported", which was the favourite one.

\section{RELATED WORK}

Approaches to provide physical interactions in virtual reality are to either simulate physical objects or to exploit the ones available from the environment. CoVR is part of a hybrid approach, which uses robotic devices to displace or render physical objects.

\section{Simulating physical objects}

Several devices have been proposed to simulate physical objects in VR, with a trade-off between the quality of the haptic rendering, especially kinesthetic feedback and the workspace size [53]. These devices are usually limited to desktop usage
$[27,23]$ but can render high quality kinesthetic feedback (over $30 \mathrm{~N}$ ) [53]. Most of these solutions provide stimulation at the scale of the hand without any regards concerning whole-body interactions. To widen the workspace, alternatives attach the device to mobile platforms [41, 24, 52, 48, 25, 40, 26, 25], which remain slow and require to be held continuously whilst moving (which is opposed to Krueger's postulate to develop unencumbered artificial realities [67]).

In contrast, several low-cost haptic devices have been proposed in HCI. Typically, wearable or hand-held devices [4, 1, 44, 8, $11,68,21,61,31,57,42,22,6,20]$ are naturally compatible with large environments. They can simulate various haptic features (weight, stiffness, shape, texture) on different body parts. However, they provide limited kinesthetic feedback and they need to be held continuously.

\section{Exploiting physical objects}

The second approach exploits directly physical objects placed in the VR arena, following Insko's postulate that passive props enhance virtual environments [37]. For instance, one solution is for each virtual object to annex a physical object with similar properties in the VR arena [32]. Another solution is to use human actuators [19] who execute a subtle choregraphy to move the physical objects at the right place and time. The choregraphy is rather costly and time-consuming. Some solutions aim to reduce the number of human actuators to move physical objects by using other users $[17,16]$ or even the users themselves [15] at the cost of reducing the number of interactive features.

\section{Robotic Shape Displays}

The concept of Robotic Shape Displays (RSD) [45] focuses on the mobile, unencumbered and untethered aspect of the human [67]. It consists of using a robot to overlay virtual objects with physical props. We distinguish the robotic system (hardware) from their trajectory generation algorithms (software):

Robotic system. Recently, several classes of prototypes aimed to instantiate the original Robotic Shape Display concept by displacing objects $[58,5,30,38,36]$ or simulating them [56] to meet the users without impairing their movements.

Drones can transport objects that users can explore [70, 39, 33] or manipulate [5] in a theoretically unlimited workspace (in practice, the workspace is limited to $2.5 \mathrm{~m}^{3}$ because of technical constraints). This approach only provides a small amount of force feedback as state-of-the-art drones can not resist to human actions. Moreover, this technology has several technical limitations including speed $(<0.5 \mathrm{~ms} / \mathrm{s})$, accuracy $(>7 \mathrm{~cm})$, autonomy and safety. Moreover, the accuracy and reliability of these systems decrease with the embedded mass of the props, which reduces the range of available scenarios with high kinesthetic feedback.

Lightweight mobile robots [30, 58, 66]) or lightweight robotic arms $[7,65,73,45,69,55,35]$ provide a medium amount of force feedback, but lack an efficiency of displacements. Mobile robots are limited in speed $(<0.5 \mathrm{~m} / \mathrm{s})$ and autonomy while robotic arms are limited to desktop usage. 
Only few prototypes provide a high amount of force feedback. TilePop [62] or LiftTiles[59] modify the floor topology with a large inflatable mat covering the surface of the VR arena. This provides high vertical force feedback and hence supports whole-body interactions below $1 \mathrm{~m}$ (eg user sitting [74]), despite slow inflation (5s) and deflation (20s) times. Relying on a robust robotic arm such as a Kuka $[38,46]$, can produce a high blocking force and hence provide large kinesthetic feedback at a body-scale, in spite of its cost. This robustness obviously goes along with strong software safety considerations around the user. These prototypes do not easily scale to VR arenas and do not let users freely manipulate a wide range of props.

Trajectory Generation. The quality of the interaction does not only depend on the hardware implementation (eg speed) but also on the algorithm to generate trajectories, especially in non-deterministic scenarios ${ }^{2}$. When the device does not know in advance which virtual object to physically overlay, it is thus necessary to (1) build a user intention model (e.g. [12]) to estimate what will be next object to overlay and (2) a path planning algorithm (e.g. [18]) to displace the robot to the target location without colliding obstacles. Only few of the above systems $[38,72,73]$ rely on these components to support non-deterministic scenarios. However, they require multiple robots $[58,65]$ or specific devices (e.g. eye-tracker [12]). In contrast, we elaborate a low-computational intention model working with common HMDs.

In summary, Robotic Shape Display systems still face multiple interaction (e.g. whole body interaction, locomotion, force feedback, free manipulation of multiple props) and technical challenges (speed, accuracy, safety, price). CoVR addresses many of these challenges and focuses on providing large forcefeedback $(100 \mathrm{~N})$. CoVR supports whole-body interactions while letting users physically unencumbered. It is designed for Gaming or Training purposes in large virtual arenas $\left(\approx 30 \mathrm{~m}^{3}\right)$. CoVR can carry potentially heavy physical props to match virtual objects the user is about to interact with, without sacrificing speed, accuracy, safety or price.

\section{DESIGN AND IMPLEMENTATION OF COVR}

When designing our Robotic Shape Display, CoVR, we primarily focused on force feedback and workspace size as design considerations (which is a challenging trade-off for haptic devices [53]) as well as speed, interaction opportunities, price and safety. We initially considered mobile robots such as [29]. However, these interfaces are limited by a compromise between force-feedback, speed and autonomy. More particularly, we decided to focus on enabling strong force feedback at a whole-body scale and allowing different body postures. We then deliberated upon a grounded solution, a 2D Cartesian ceiling-mounted robot, which can be integrated into a roomscale arena ${ }^{3}(4 \times 4 \times 2.5 \mathrm{~m} ; L x W x H)$. The advantages are speed, accuracy, force-feedback, while allowing to move potentially

\footnotetext{
${ }^{2}$ Non-deterministic scenarios consist of scenarios where multiple virtual objects are available at the same time, and the user is free to interact with any of them. The system does not know in advance which one to physically overlay.

${ }^{3}$ The robot can be mounted on the ceiling or on an external truss structure (triangle aluminum Global Truss) as shown in Figure 2.
}

heavy physical objects without the embedded mass affecting its displacements.

Another important consideration was the number of degrees of freedom (DoF) of the robot $(X-Y$ planar motion, $Z$ elevation, $W$ rotation around $Z, 6$ DoF Robotic arm...). When dealing with robotic interfaces, a trade-off between price, complexity and interaction possibilities can be drawn. We realised that 2D planar motion carrying a modular structure already allows quite a large variety of scenarios while keeping a low technical complexity and cost. However, our chosen architecture can be extended with additional DoFs (for instance, by attaching a 6DoF Kuka robotic arm; see section Discussion).

In the following sections, we describe the main components of the final ${ }^{4}$ version of our system. We then evaluate CoVR in a technical evaluation validating its control through a userintention based algorithm.

\section{Robotic system}

Robot. CoVR relies on a 2D Cartesian ceiling robot (Figure 2), actuated with DC Motors (Dunkermotoren 55x30, KPL43 gearbox, $1.81 \mathrm{Nm}$ torque for $X$-axis, Dunkermotoren $63 \times 55$, KPL57 gearbox, $9.75 \mathrm{Nm}$ torque for Y-axis) trough a pulleybelt mechanism (Figure 2). We chose a pulley-belt mechanism because it is simple to implement and can easily be scaled to larger VR arena. The robot moves a $15 \times 15 \mathrm{~cm}^{2}$ carriage on which is attached a modular structure (see below).

The robot is controlled in speed with a Roboclaw $2 \times 30 \mathrm{~A}$ V5E motor controller and AEAT-601B-F06 encoders, mounted on a custom-designed 3D-printed support. The Roboclaw controller is connected to an Arduino MEGA 2560 microcontroller. It provides closed loop control with a $P I V^{5}$ scheme. The total price of the robot (motors, rails and pulley-belt) is under 1500euros.

Speed. The speed of the robot depends on the distance to travel. For large distances $(>1 \mathrm{~m})$, the speed is over $\mathbf{1 . 1} \mathbf{~ m} / \mathbf{s}$,

\footnotetext{
${ }^{4}$ We used an iterative process to design CoVR. At each iteration, we improved key features such as robustness, accuracy, speed, safety, while widening the interaction possibilities.

${ }^{5}$ Proportional position loop Integral and proportional Velocity loop
}

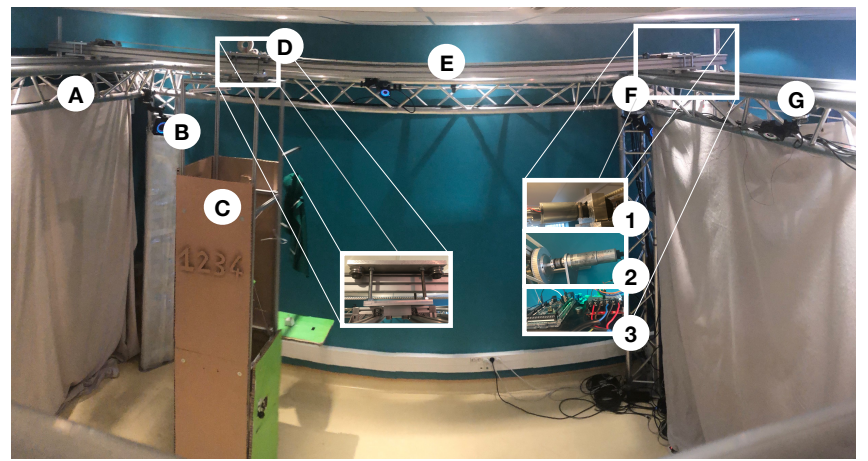

Figure 2. Top isometric view of CoVR setup: (A) Structure; (B) Skeleton, modular column-like structure to attach props and panels; (C) CoVR panel; (D) Carriage; (E) $X$-axis rail; (F) 1: $X$-Pulley-belt system and Motor, 1: $Y$-Pulley-belt system and Motor, 3 - Electronics (Arduino and RoboClaw); (G) $Y$-direction rail. 
which is approximately a normal human walk speed. For small distances $(<80 \mathrm{~cm})$, speed is about $0.5 \mathrm{~m} / \mathrm{s}$, which remains faster than current mobile solutions (e.g. [58, 30]).

Noise. At full speed, CoVR's average noise is $55 \mathrm{~dB}$ average (max: $65 \mathrm{~dB}$ ).

Weights and Forces Capabilities. The carriage can support a total weight of $800 \mathrm{~N}$ vertically $(\approx 80 \mathrm{~kg})$ and $1000 \mathrm{~N}$ horizontally. The embedded mass the carriage can support is large enough to support a human lying on it or even to be pushed by it (Figure 1-A,B) without causing any damage to the structure. The system can also provide high traction force to pull the user (-C) or even transport her (-D).

\section{Column}

A column-like modular structure (Figure 3-A) is attached to the moving carriage. Different surfaces in arbitrary positions, shapes, orientations ${ }^{6}$ and sizes can be attached using a simple clamping mechanism. It is similar to stage designing in real theatres [49], where a limited number of decors can quickly be replaced. Another advantage is to easily support DIY: the stage designer can use cardboard or props with different mass, textures and shapes that users can freely manipulate at different heights of the column. The positions and shapes of the physical objects are then communicated to the VR designer in a calibration phase. In summary, the column has been designed to be flexible enough to support a wide range of interactions. Figure 3-B and -C show two examples of implemented columns. The section Interactions and Demo Applications detail interactions with these columns.
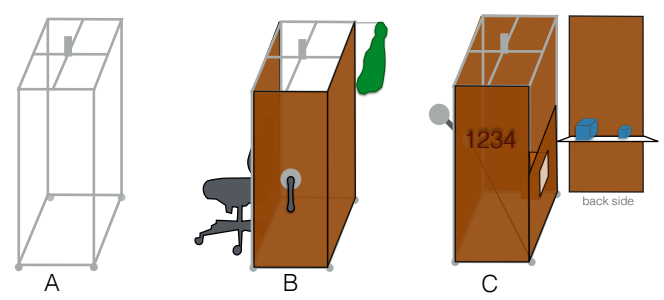

Figure 3. Column design. (A) Modular structure attached to the 2D ceiling robot to provide a wide variety of surfaces and props. (B) The 3side column used in the user study with a chair (left), a cylinder attached to a spring virtually representing a broom (front), a large cardboard simulating a wall and piece of fabric representing a ghost (right). (C) A 4-side column implemented with a lever attached to the structure with an elastic (left), haptic code made in cardboard and glue (front), and a tray with a large and small cube (back) to insert into the locker (right).

\section{Display and Tracking}

We use the Oculus Rift S [2] HMD because it is not sensitive to occlusion problems and it allows interactions under or even in the column (Figure 8). We used Unity3D to create virtual scenes. It centralises the communication and synchronisation between different components though plugins (SteamVR, Arduino/Roboclaw). In particular, the SteamVR plugin asset [3] is used for the Oculus communication and the Uduino package [63] for fast prototyping between Arduino and Unity.

\footnotetext{
${ }^{6}$ This feature made the $W$ rotation partially redundant.
}

\section{Safety}

As users are invited to move around an active large-scale mechanical system, safety measures had to be established. These were planned on several levels, from the structure conception to the motions around the users during interactions.

Carriage: One risk is the fall of the carriage. The carriage can support both larger axial $(800 \mathrm{~N})$ and radial $(1000 \mathrm{~N})$ forces than those required for the envisioned scenarios. A security coefficient of 2.5 was introduced for elastic deformation calculations in the conception process.

Column motions: Hardware, software and electronic emergency stops are implemented. The carriage motion is restricted on both ends with spring-based mechanical stops. The software stops the motors when the column is within $2 \mathrm{~cm}$ of these limits. The controller electronically shuts down when the motor's current exceeds 5A. More importantly, the column immediately stops if the user is not tracked for more than $0.5 \mathrm{~s}$. Finally, the game master has a manual emergency stop button that turns the system off, keeping it electrically grounded to avoid potential shocks. Finally, given the power and speed of the robot, it is important to ensure the column will not accidentally physically collide with the user. We thus developed an algorithm to generate the robot trajectories.

\section{Robot Motion Control}

We present a model to control the robot displacements ${ }^{7}$. While trajectories are easily generated by the Cartesian structure (XY displacements), the algorithm inputs for scenarios involving multiple objects of interest need to be defined and safety measures around the user need to be implemented. The main idea is to attach the robot to a virtual proxy (a ball with mass and gravity) with a spring-damper model. The ball's displacements depend on (1) the user's location to avoid collisions, (2) the user intentions and (3) the progress of the scenario to attract the ball towards the objects users are most likely to interact with next. A key contribution regarding our trajectory generation model is the elaboration of a low-computational user intention model working with common HMDs. We now detail our approach to generate trajectories.

Trajectory generation. Each virtual object of interest $i$ within the scene gets a weight $W_{i}$ which depends on its likeliness to be interacted with next. The virtual proxy (ball) and CoVR command position $\operatorname{CoVR}(x, y)$ is hence a weighted average of the positions of each object of interest:

$$
\operatorname{CoVR}(x, y)=\frac{\sum_{i=1}^{N} W_{i} *\left(x_{i}, y_{i}\right)}{\sum_{i=1}^{N} W_{i}}
$$

where $N$ is the number of virtual objects of interest (VOI) in the scene, $\left(x_{i}, y_{i}\right)$ the cartesian coordinates of the VOI $i$ and $W_{i}$ its weight, estimated given a user intention model (see below). A virtual spring between the proxy and the command position is then defined, and the according spring force is applied for the proxy to reach this position. We use Unity3D's physics engine to automatically generate the proxy trajectories to reach a target. The target position is not necessarily the

\footnotetext{
${ }^{7}$ The algorithms, models, scripts and user data are available on this repository: https://www.bouzbib.com/CoVR/
} 
position of a virtual object. If the scene contains two objects of same interests, CoVR will automatically place itself between these two objects' positions, hence the displacement when one becomes the chosen object of interest is minimised and CoVR is more likely to reach it prior to user interaction. As the proxy is also attached to the CoVR, its resulting motion takes naturally into account the robots speed limitations.

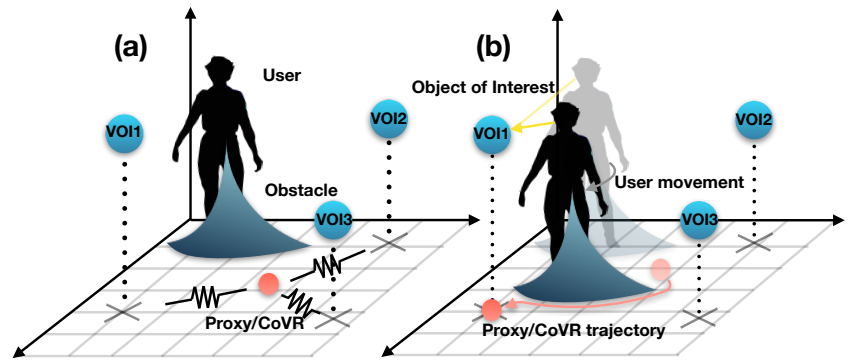

Figure 4. Control algorithm relying on a physical model: (a) The virtual proxy of the physical CoVR column is connected to all virtual objects of interest (VOIs) with weights depending on the users' intentions to interact with them. The user and other forbidden zones are covered by a rigid cone-like obstacle to be repulsive. (b) Whenever the user is about to interact with a VOI, the proxy/CoVR move towards it, while naturally avoiding obstacles (e.g the user).

We also created virtual obstacles to cover all forbidden areas in the arena (user, external people, furniture). Indeed, we designed a cone-like rigid shape that we attached to the user's position as shown in Figure 4. The size of the cone (diameter $=90 \mathrm{~cm}$ ) was chosen to avoid collisions even if the users' arms are open. Thanks to the contact mechanics and gravity in Unity, the proxy is naturally pushed and rolls away from the obstacle. The obstacle's curvature ensures a smooth deceleration of the proxy when this latter is getting to close to it. The radius of the obstacle decreases $(20 \mathrm{~cm})$ when the user comes near a object of interest, so the proxy is not pushed away.

\section{User-intention model}

We elaborated a user intention model to support nondeterministic scenarios, i.e. scenarios where the system does not know beforehand which object to physically overlay. The model inputs are the positions of the virtual objects of interest (VOI) as well as the available data from the users' apparatus: the HMD's position and orientation. It hence does not require additional hardware such as eye-tracker or finger/hand tracker.

We defined the total weight $W_{i}$ of the VOI $i$ to be a function of the user's distance $D$ to a VOI, and her orientation $(O)$.

$$
\begin{aligned}
& W_{i}(d, \theta)=\omega * D(d)+(1-\omega) * O(\theta) \\
& W_{i}(d, \theta)=\omega * \frac{1}{1+d}+(1-\omega) * e^{(\cos (\theta)-1)}
\end{aligned}
$$

where $\omega$ is the contribution of the distance over the orientation. $D(d)$ and $O(\theta)$ 's ranges are between 0 and 1 , hence $W_{i}$ 's range is from 0 to 1 too. $O(\theta)$ is equal to 1 whenever the user's HMD orientation is colliding with any surface point of the VOI's mesh, and is decreasing exponentially whenever the user's orientation moves further away. On the same principle, $D(d)$ is equal to 1 whenever the user is close to a target, and decreases with the same regards ${ }^{8}$.

\footnotetext{
${ }^{8}$ We also increase the stability of the column in the vicinity of the VOI. When $W_{i}>0.8, W_{i}$ is rounded to 1 , typically when an object is
}

\section{Scenario-based model}

Depending on the progress of their scenario, designers can estimate the prior probability of an object to be interacted with: in a basketball game for instance, the user is more likely to interact with the ball first than with the hoop. We let the possibility to designers to define their own scenario-based model by refining the estimation of $W_{i}$ :

$$
W_{i}=P_{i} \times W_{i}(d, \theta)
$$

where $P_{i}$ is the prior probability of the VOI $i$ to be interacted with from the progress of the given scenario. We will discuss the use of these probabilities in the Discussion section of our Technical Evaluation below.

\section{TECHNICAL EVALUATION}

The primary aim of this technical evaluation is to determine the $\omega$ parameter of the user intention model, i.e. the optimal contribution of the distance over the orientation to estimate which object of interest is more likely to be interacted with. We are also interested in studying CoVR's success rate as a function of the number of objects of interest (distractors) within the scene. Indeed, we anticipated that the performance of the user intention model and the value of $\omega$ depend on the number of distractors within the scene. Finally, we want to confirm that CoVR's speed is sufficient enough to reach a virtual object of interest even when the user does not have a decision to make (number of VOI = 1).

We first perform a data collection over a panel of users to better understand how intentions can be quantified as a function of both distance and orientation. We then perform multiple physical simulations to find the best $\omega$ parameter that matches users' behaviors.

\section{Data Collection}

Participants and Apparatus. 6 participants ( 3 male, 1 lefthanded) aged from 26 to 32 (average $=28$; std $=2.0$ ) volunteered for this experiment. All participants were familiar with VR and were asked to wear the Oculus Rift S. Users also wore Optitrack markers on their dominant hand. The Oculus headset was also equipped with Optitrack markers, for an accurate tracking in space. The virtual scene was created using Unity3D game engine.

\section{Experimental Design}

Task and Stimuli. We considered an exploratory task, such as the ones users would perform in games, i.e. users take their time, observe the decors, avoid virtual obstacles and face their objects of interest whenever interacting. To replicate these game features and to capture the corresponding users' behaviors, we created an empty scene where virtual numbered balls appear simultaneously at random locations with random orientations (see Figure 5). Instructions are written on the walls surrounding the users, and tell them to touch a given numbered virtual target. Users are then asked to face the targets whenever touching them.

at less than 20 degrees from the user's HMD direction or when the object is at a distance below $20 \mathrm{~cm}$ from the user. It allows for CoVR to stay at the closest VOI as long as the user remains in its vicinity. 
Conditions. In this experiment, we control the number of distractors within the scene from 0 to 4 (number of balls is from 1 to 5). This allows us to understand the performance of CoVR over the number of available VOIs. The minimum distance between two targets is their diameter $-10 \mathrm{~cm}$ (eg they cannot overlap) and they cannot appear at the user's location. As long as the user does not touch the target ball, nothing changes in the scene. As soon as the target ball is interacted with, another condition starts.

Design. We used a within design. All participants tested all five conditions ( $0,1,2,3,4$ distractors). The order of appearance of each condition was randomized within the blocks. Participants performed 10 blocks. The duration of the experiment was about 12 minutes per participant (std $=2.6$ ). In summary, the experimental design is: 6 participants $\times 10$ blocks $\times 5$ conditions $=300$ trials.

For each trial, we measure the users' position and orientation at each frame, with a frame rate of $75 \mathrm{fps}$.
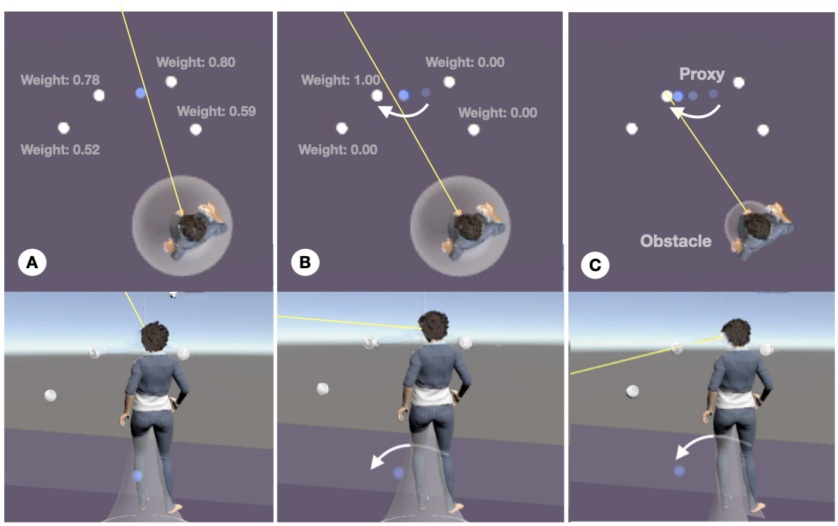

Figure 5. Technical Evaluation "Simulation" Virtual Scene example after the Data Collection. (A) User looks for the target (according to the walls' instruction). Weights change according to her position and orientation. (B) Intention Detection: User chooses a target and its weight goes to 1. (C) Trajectory: The proxy (blue ball) moves accordingly with the centroid of all the objects' of interest's weights towards the chosen one (weight $=1$ ), while avoiding the user obstacle. When the proxy reaches the chosen ball, the user obstacle size decreases.

\section{Parameter Fitting}

We used the data collection to replicate the users' displacements into a Simulation virtual scene. The robotic system physically moved accordingly with our "user intention model" (section above). Each simulation corresponded to a different $\omega$. We simulated all the data from the 6 participants (i.e. including the 5 conditions). We first performed a broad exploration of $\omega($ step $=0.25)$ and then refined it to find the optimal one for each condition (number of distractors in the scene). We tested 13 parameters over 6 users, which resulted in more than 17 hours of simulation.

Our main measurement was the success rate of CoVR reaching a VOI before the user, i.e. when CoVR's distance to the target was below its diameter $(10 \mathrm{~cm})$ when the user was touching it.

\section{Results}

Success Rate. Figure 6 shows the success rate as a function of $\omega$ and the number of distractors. The success rate is approxi- mately $100 \%$ (only $1 / 300$ targets missed) when there is only one VOI in the scene, indicating that the system is at least as fast as the participant when the target position is known (i.e. the system does not rely on the users' intention). The results also confirmed that the success rate decreases with the number of distractors. Figure 6 also shows that we obtain the best average success rate (80\%) with $\omega=0.175(\mathrm{CI}=14 \%)$ regardless of the number of distractors. Success rate remains above $80 \%$ up to 2 distractors.

We also note that the success rate per user decreased with the time spent in the experiment (88\% success for a $14 \mathrm{mn}$ experiment vs $74 \%$ for an $8 \mathrm{mn}$ experiment).

Target distance. We measured the average distance between the carriage and the target centres when the user was colliding with the virtual target. The average distance among all the trials is $1.8 \mathrm{~cm}(95 \% \mathrm{CI}=0.33 \mathrm{~cm})$ demonstrating the repeatability of our implementation.

Detection time. We also measured the time difference between the target's weight reaching 1 and the user colliding with it. Results show that this detection time does not depend on the distractors, with a $7 \mathrm{~s}$ average (std $=0.6 \mathrm{~s}$ ) and a $96 \%$ accuracy. We note that if the detection time is below $4 \mathrm{~s}$, it results in a failure of the overlaying, as CoVR struggles to get around the user (especially the obstacle) and place itself properly.

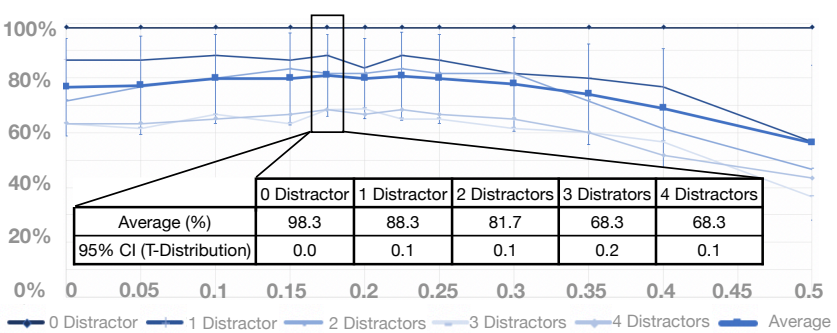

Figure 6. Success Rate of CoVR reaching the chosen VOI prior to the user interaction, function of $\omega$ and the number of distractors. Error bars indicate $95 \%$ confidence interval with a T-Distribution. The table shows the Success Rate with the optimal parameter, $\omega=\mathbf{0 . 1 7 5}$, as function of the number of distractors.

Number of users collision. No collision between the user and CoVR were noted during the simulations.

Accuracy. Finally, we measured the distance between the virtual proxy and the physical column. The mean distance over all users and conditions is $0.94 \mathrm{~cm}(\mathrm{CI} 95 \%=0.99 \mathrm{~cm})$, which ensures they share the same trajectory, and hence a safe user environment around CoVR.

\begin{tabular}{|c|c|c|c|c|c|}
\cline { 2 - 6 } \multicolumn{1}{c|}{} & 0 Distractor & 1 Distractor & 2 Distractors & 3 Distrators & 4 Distractors \\
\hline Average $(\mathrm{cm})$ & 0.6 & 0.9 & 1.0 & 1.2 & 1.0 \\
\hline STD $(\mathrm{cm})$ & 0.4 & 0.7 & 0.8 & 1.0 & 1.0 \\
\hline
\end{tabular}

Table 1. Accuracy, measured by the distance between CoVR and the proxy, with $\omega=\mathbf{0 . 1 7 5}$.

\section{Discussion}

This evaluation tested CoVR in an uncontrolled environment, with random locations and orientations for each target and distractor and a user-intention based model. Despite this environment, our system had a high success rate $(>80 \%)$ with 
three virtual objects of interest while preserving the user's safety (no collision). Multiple directions can be envisioned to increase this success rate in non-deterministic scenarios.

Adding a scenario-based model. According to the Equation 4, we can add "prior probabilities" to the different VOIs, depending on the progress of the scenario. After selecting our optimal parameter $\omega=0.175$, we ran the robotic simulation by adding to the actual target a $75 \%$ probability to be interacted with. The distractors were hence splitting the remaining $25 \%$ of interaction probability. The results are summarized in the Table 2 and confirm that adding a scenario-based model improves the prediction with a success rate higher than $93 \%$ even with four distractors.

\begin{tabular}{|c|c|c|c|c|c|}
\cline { 2 - 6 } \multicolumn{1}{c|}{} & 0 Distractor & 1 Distractor & 2 Distractors & 3 Distrators & 4 Distractors \\
\hline $\begin{array}{c}\text { Average \% (95\% Cl T- } \\
\text { Distribution) }\end{array}$ & $98.3(1.8)$ & $95.0(2.4)$ & $93.3(4.4)$ & $96.7(3.5)$ & $93.3(3.5)$ \\
\hline $\begin{array}{c}\text { Average Distance to Target } \\
\text { (STD Distance, in cm) }\end{array}$ & $0.4(1.4)$ & $0.7(1.7)$ & $0.6(1.6)$ & $1.0(1.8)$ & $1.2(2.2)$ \\
\hline
\end{tabular}

Table 2. Success Rate and Distance to Target with $\omega=0.175$, and a $75 \%$ probability to be interacted with added on the target.

Assigning multiple VOI to the same Physical position. Thanks to its size and shape, CoVR can contain multiple objects on different panels and at different heights. We can take advantage of this feature to assign multiple virtual objects of interest to the same physical location, hence reducing the amount of CoVR displacements and the risk of spatial mismatches.

Adding visual effects. When a spatial mismatch is likely to occur, literature usually proposes to cater for it with visual effects [19] or dynamic redirection techniques [?, 50, 9]. These respectively distract the users and give spare time to the robot to reach the target location or dynamically correct the users and CoVR's interaction positions.

\section{INTERACTING WITH CoVR}

The technical evaluation showed that CoVR is able to move at a sufficient speed to follow users at a natural walk speed.

When no interaction is required, CoVR remains out of reach and the users can wander in the whole arena. CoVR thus does not interfere with users' natural behavior. Letting the users truly walk (instead of using a metaphor for locomotion) reinforces the immersion [64].

When interactions are required, a key aspect of CoVR is to allow interactions involving strong kinesthetic feedback at a body scale. We distinguish two main uses of CoVR: static use where users transmit forces when interacting with the column (e.g. exploration, manipulation) and dynamic use, where users are receiving forces enabled by CoVR's displacements during the interaction (e.g. leading through forces, transport). We now detail these two uses of CoVR.

\section{Static Use of CoVR}

Hand exploration: Hands remain the primary body part for exploring the world and the most sensitive one. Users can probe objects directly with their bare-hands. As such, interactions are not limited to one finger: surfaces can be realistically touched and their texture fully felt with the whole hand. In particular, users can interact with the palm, which contributes to a sense of tangible presence, as it enables kinesthesia on top of tactile cues [47]. Moreover, the explored surface can be large and not limited to a specific orientation or shape. For instance, users can perform large hand movements to find a specific tactile pattern on a wall for instance (Figure 7-A).
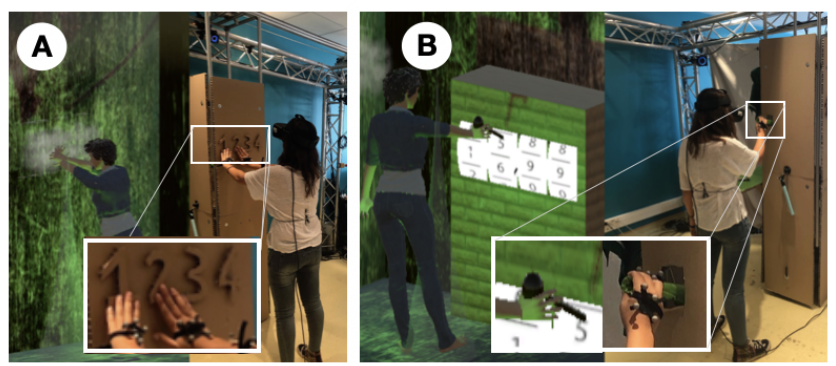

Figure 7. (A) Tactile Exploration: The user tactilely explores large surfaces, for instance, to find a hidden code over a human-sized wall. (B) Directed Manipulation: The user pulls a lever which is attached to CoVR with an elastic, letting it a single degree of freedom, providing a mechanical manipulation of props.

Whole-body interactions: Users can apply strong forces with any part of their body: users can lean on a fixed wall (Figure 1 - B), push hard on it with their hands or shoulders (Figure 10) or even kick it. CoVR is rigid and robust enough to remain still during all of these interactions.

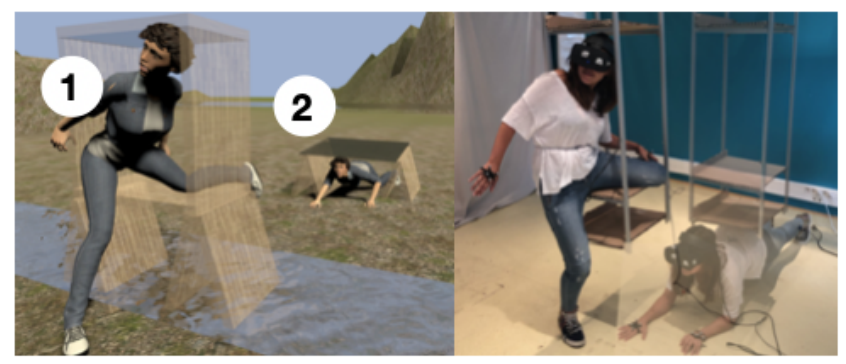

Figure 8. Postures; The user (1) goes through an obstacle with constrains below and beneath her (2) or crouches.

Postures: CoVR also supports a variety of users' postures with interactions at different heights such as crouching under a table, going through obstacles with physical props both below and above the users (Figure 8), sitting on a chair $[74,60]$ or climbing a stair to reach a high target (Figure 10).

Manipulation: Manipulation of real objects and passive props improves interaction fidelity $[51,37]$. CoVR enables different types of object manipulation:

- Free manipulation. CoVR can carry untethered objects which users can grab and freely manipulate. A large variety of samples (Figure 9-B) of any textures is possible, as long as dimensions and weights are compatible. Thanks to the CoVR's grounding and high motor torques, it can carry large masses without compromising its speed or accuracy.

- Contact. Objects can also be manipulated to interact with each other. For instance, in Figure 9-C, the big cube does not physically fit in the locker. The user hence needs to find a smaller one. 
- Directed manipulation. Users can interact with objects tethered to CoVR. Its structure allows for mechanical manipulation of objects and for users to actuate them. For instance, in Figure 7-B, the user actuates a lever mounted on the column, simulating a slot machine. By attaching objects on CoVR's skeleton, mechanical manipulation with multiple numbers of degrees of freedom is possible.

A single physical object can overlay multiple virtual ones of similar primitives [32]. Instead of using visual effects such as [10], CoVR physically moves a single prop to overlay multiple virtual ones. For instance, one physical door can overlay three virtual ones (Figure 9-A). These mappings were previously seen in the literature [30, 29].
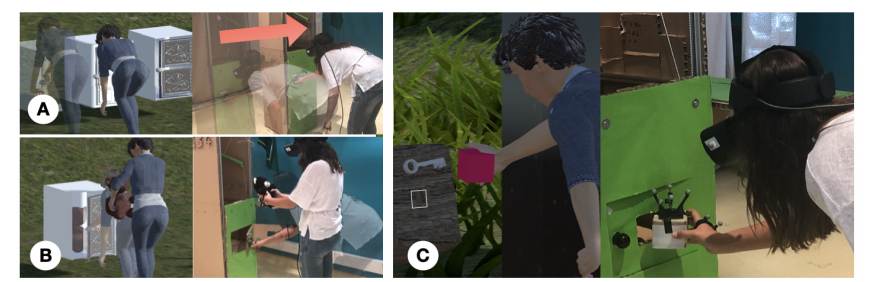

Figure 9. (A) Directed manipulation; User opens three virtual doors but only a single physical one, cut through a panel cardboard. (B) Free manipulation; User finds a teddy bear. (C) Free manipulation and Contact; The user manipulates a cube which is too big to fit in the locker. She realises she needs to find a smaller cube.

\section{Dynamic Use of CoVR}

In the previous section, CoVR was motionless during the interaction (static). The following interactions require the system to move in the user's vicinity (dynamic).

Receiving Physical Contact: CoVR can physically touch the users and produce impact force feedback [66]. It is thus initiating the haptic interaction, instead of the user. As receiving an interaction might be surprising in VR, we recommend attaching props at a distance from CoVR's main skeleton, to produce light impact forces. For instance, a fabric $(60 \mathrm{~cm}$ away from the main skeleton) can lightly brush the users to simulate the crossing of a ghost (Figure 12) through them. Users can also be touched by a virtual agent trying to catch their attention, providing a sense of physical presence [43, 34].

Leading through forces: Users can be led by CoVR through body-scaled tension and traction forces. For instance, in Figure 1-C, the user physically holds a cylinder attached by a spring to the column, virtually represented by a broom, which provides her with a large force-feedback and leads her the way in the virtual environment. She is pulled by the broom when CoVR moves. Another example is inspired from [17], involving a fishing pole where the line is attached to the column. The motion of the column creates the illusion of a fish biting.

Transport: Finally, CoVR mechanical properties open up a new range of interactions in VR. Indeed, CoVR can transport the users. For instance, it can move a chair with a sitting user to a different location (Figure 11) as CoVR can handle large embedded masses. We envision other scenarios transporting the user, such as Wind-surfing or Water-skiing [71].

\section{DEMO APPLICATIONS}

We created a two-scene demo application to demonstrate the interaction possibilities offered by CoVR. It relied on the 3-side column illustrated in Figure 3-B and involved 5 interactions, 7 virtual objects but only 5 props. In the following subsections, user interactions will be displayed in bold while the motions and CoVR's interactions will be displayed in italics.

\section{Escaping the Room}

We created a first scene where the users need to escape a room.

\section{Reaching for the Light}

First, Bob is in a dark room where the only thing visible is a light bulb, at a $2.5 \mathrm{~m}$ height, in a small cupboard. Bob hence climbs in the cupboard to touch the bulb, which then turns on the lights. In the physical world, he hence goes into CoVR, which remains still and touches the top of CoVR's skeleton.

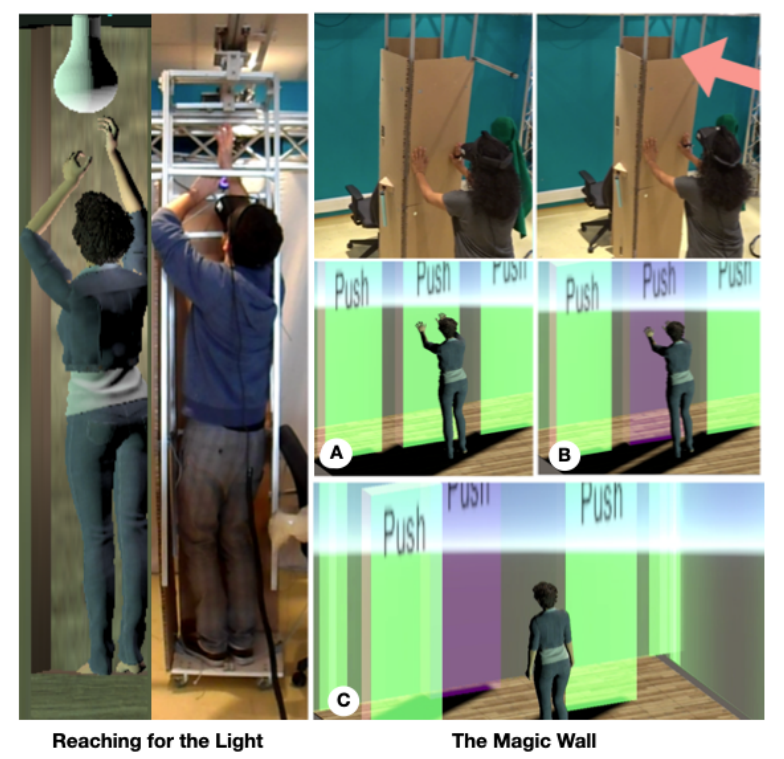

Figure 10. "Escaping the Room". Reaching for the Light: User climbs in the cupboard to reach the virtual light bulb; The Magic Wall: (A) User chooses a wall. CoVR moves accordingly with the user's intentions. User pushes on the wall. We note that none of the users touched the "ghost" by accident during the experiment. (B) The wall remains static, and changes color to encourage the user to maintain contact. (C) After 10seconds of maintained contact, the Magic Wall moves, giving the user the impression of having pushed it herself.

\section{The Magic Wall}

Bob then sees a carpet with the words "Start". Once he reaches it, three walls appear. A sign informs him he needs to push them. Bob chooses a wall, but can change his mind and pick another one if he wants. He then has to maintain contact and keep pushing for 10 seconds. The walls' color changes from green to red (accordingly with the timer), to indicate Bob he needs to keep pushing and that a maintained contact is needed.

When the walls appear, CoVR hence uses the users' intentionsbased algorithm in order to place itself at the chosen wall. When Bob pushes a wall, CoVR remains static. Once the timer is finished, CoVR steps backwards, which gives Bob the impression of having pushed the wall himself. 


\section{Travelling in the Clouds}

After pushing the walls, dust starts flying around Bob, who is then teleported in a forest.

\section{The Magic Broom}

The user now sees a magic broom. He holds it tightly, and is now pulled by CoVR, through the forest to the clouds. CoVR pulls the user with a strong force-feedback, as the broom is actually a cylinder attached to CoVR with a string and a spring (see Figure 1 - C).

\section{Moving in the Clouds}

Once the travel is over, a "Continue" panel appears. When Bob touches it, a chair appears. Bob then sits comfortably in the chair, and CoVR transports him through the clouds.

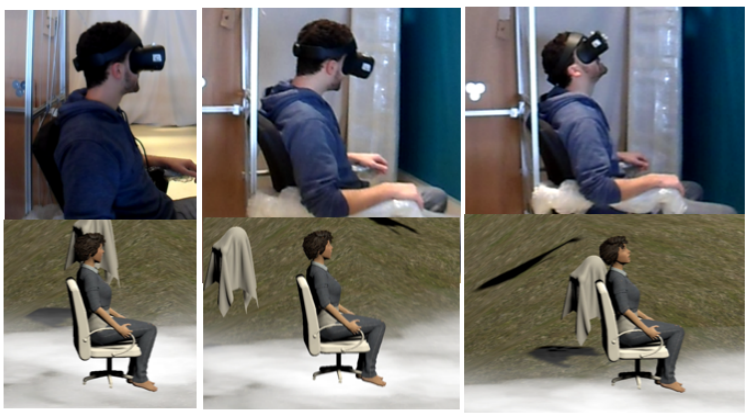

Figure 11. Moving in the Clouds: User is sitting in a chair and physically transported through the clouds.

\section{The Ghosts}

The user, in the clouds, is now surrounded by ghosts. He then sees a halo, in which he decides to go into. When he reaches it, he then sees a huge ghost about to go through him. Bob remains still while CoVR initiates the interaction by brushing his head with a piece of fabric.

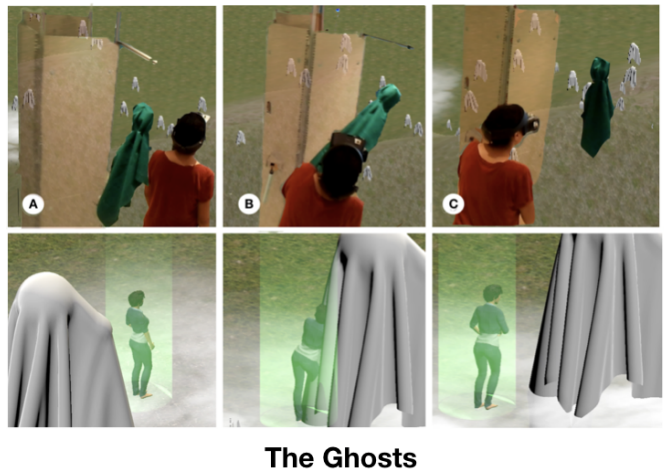

Figure 12. The Ghosts: (A) User enters the halo. (B) A piece of fabric lightly brushes the user's head. (C) The ghost flies away.

\section{USER STUDY}

The goal of this study is three-fold: (1) validating the implementation of CoVR, (2) investigating how users experience (i.e. apply and receive) strong forces and (3) collect feedback on the interactions of the demo application.

Participants. 8 participants (4 male) aged from 22 to 30 (std $=2.8$ ) volunteered to test the demo application. 4 of the participants were familiar with VR technologies, 2 had only tried VR once and the remaining 2 had never experienced VR.

Procedure. Participants were informed they were going to interact with physical props and were asked not to rush within the scene. They were asked to wear an Oculus Rift S HMD as well as Optitrack markers on their dominant hand. They all were introduced to CoVR and saw it moving beforehand. A game master was present during all the experiments, to ensure the participants' safety and activate some of the interactions. After the experiment, participants filled a Likert-scale questionnaire about their enjoyment on each demo interaction and then participated in a semi-structured interview. They gave approximately 20 minutes of their time.

\section{Results}

\section{Quantitative results.}

Participants ranked their global enjoyment with a $6.0 / 7$ grade $(\mathrm{std}=0.5)$.

Favourite interactions. Users were asked to choose their two favourite interactions in terms of enjoyment, among the five that were provided. $62.5 \%$ of the participants said their favourite interaction was the transport, while the remaining $37.5 \%$ preferred the magic broom (being pulled). The second favourite interactions were evenly split between pushing walls, the magic broom, transport and being gone through by ghosts.

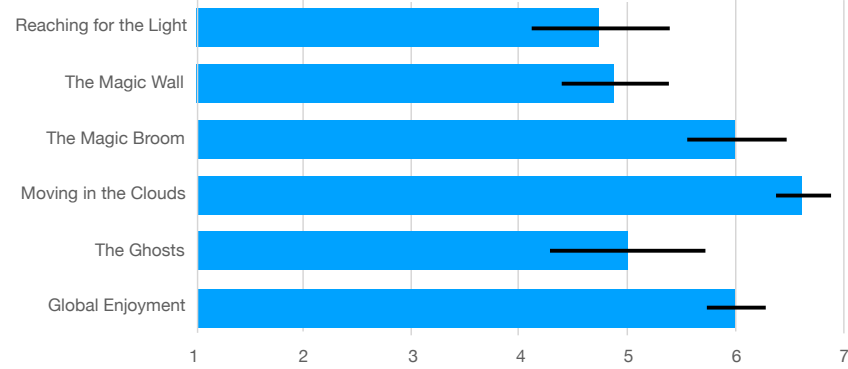

Figure 13. Enjoyment results per interaction, ranked on a 7-point Likert scale - 1 indicates "not enjoyable", 7 indicates "very enjoyable". Error bars indicate the standard deviation of the grades in the users' panel.

Force-feedback. All of the participants ranked the force they applied (wall) or applied to them (broom) compared to their maximum force on a 7-point Likert scale $(1=$ pretty soft, $7=$ very hard). The forces they applied to the walls was ranked with an average of $5.5(\mathrm{std}=0.75, \min =5 / 7, \max =7 / 7)$ while the force applied to them with the magic broom was ranked with an average of 6.1 (std=0.64). In particular, $87.5 \%$ of the participants $(7 / 8)$ ranked the force applied to them with travelling with the magic broom between 6 and 7/7 (the last participant attributed a 5/7 grade).

Spatial Mismatches. None of the participants experienced spatial mismatches, even with the non-deterministic scenario involving multiple doors.

Apprehension. The participants ranked their fear of being around a moving platform with a Likert scale $(1=$ not scary at all; $7=$ frightening). The average fear was $3.6 / 7(\mathrm{std}=1.5) . \mathrm{P} 2$ (expert VR user) told us that he would have liked to have noise cancelling ear-puffs and ranked his scare with a 6/7 grade, as 
the noise was keeping him from being fully immersed. All of the non-expert users ranked their scare with a 2 or 3/7 grade and dove into the VR environment without apprehension.

\section{Qualitative results.}

Whole-body Interactions. In our semi-structured interview, we discussed the users' game preferences. All of the participants told us that they prefer whole-body interactions in exploration games, where performances do no matter. They all informed us they enjoyed our game and the interactions it provided, and were mostly surprised to be pulled by the broom or transported.

Force-feedback. They were especially surprised by the force provided by the broom, as it was the first dynamic interaction they were experiencing. P5 said that she was afraid of heights in the virtual scene, so when the broom started pulling her, she felt quite stressed out. P4 told us she enjoyed the use of passive props and direct manipulation [14].

Future Interactions Opportunities. We asked participants to give us feedback on interactions they would like to experience in VR with CoVR. Two external expert users told us that they would enjoy climbing on a wall. P4 mentioned virtual escape game, where she could truly benefit from passive haptics, manipulate objects and feel force-feedback. P2 and P6 suggested war games, where they could lean on the walls to get some rest, or hide from enemies. P7 added he would enjoy having more modalities involved, for instance he would appreciate having a sensation of wind when climbing (on a stair or else) to increase his immersion.

\section{Discussion}

We now summarize and discuss our main findings.

CoVR implementation. The experiment confirmed the robustness of CoVR as it did not show any failure during the experiences: Our robotic system applied or received strong forces by the participants without damage. Moreover none of the participants experienced collision or spatial mismatches while they were freely walking in the entire room-scale arena thanks to our trajectory generation algorithm and more particularly our user-intention model.

Experiencing strong forces. The experiment also revealed the benefits of robotic interfaces and more specifically robotic shape displays providing strong forces. Indeed, participants spontaneously applied 5.5/7 of their maximum forces when pushing on the walls. One participant reported having applied "very hard" forces (7/7). Moreover, participants perceived the strong (6.1/7) tension forces when interacting with the broom and enjoyed them (second favourite interaction, and an average of 6/7). Seven participants reported having received "very strong" forces $(>6 / 7)$.

Transporting the user. The favourite interaction was "transport" (6.6/7) where a user was sitting on a chair moving in the VR arena. This interaction requires both a large arena and a robotic system able to displace heavy embedded masses, which are unique features of CoVR.

In summary, this experiment revealed that whole-body interactions involving strong forces (applying forces, receiving forces or embedding heavy masses) are a promising direction for future Robotic Shape Displays.

\section{CONCLUSION AND FUTURE WORK}

We presented CoVR a novel Robotic Shape Display for roomscale VR arena providing whole-body interactions and strong force feedback. We also proposed a low-computational user intention model compatible with common HMDs to support non-deterministic scenarios. The technical evaluation and the user study demonstrated the feasability of the approach, its usability and the relevance of interactions involving strong forces. While CoVR addresses several interactions and technical challenges, we see several directions for future work.

Adding multiple columns. A main limitation of our current setup is the use of a single column in the VR arena. One approach consists of mounting additional 2D Cartesian robots on the sides of the VR arena to control horizontal columns. Another one is to add a second ceiling robot (the robots can share the rails). These two approaches limit the work-area of the additional columns but appropriate control strategies can optimize trajectories and augment interaction possibilities, especially with multiple users (see below).

Combining multiple RSDs. Our approach is compatible with previous Robotic Shape Display solutions. For instance, we envision a VR arena combining CoVR with a swarm of mobile robots such as $[58,30,66]$. These ones can collect objects on the ground and bring them back to CoVR. Our CoVR's trajectory generation algorithm remains valid in such configurations. More DoFs could also be integrated to CoVR by coupling it with a Kuka robotic arm or a Snake Charmer [7] interface.

Augmenting I/O capabilities. We will investigate how additional capabilities can improve user experience. For instance, it would be interesting to augment a column with sensors (e.g. touch input, force sensors, proximity sensors, etc.).Adding a depth camera could enable the detection of untracked moving bodies, such as an unexpected pet in the VR arena. Haptic stimuli can be expanded to vibrations, sliding, textures, temperatures, or to shape changing illusions. For instance, heat-lamps or wind-blowers could also be integrated [54].

Collaboration. Finally, our system is currently designed for a single user interaction. We plan to investigate remote-presence interaction: a second identical structure can for instance be assembled in another room. Users in each room can interact with different VOIs, share mutual physical contact or collaboratively manipulate objects $[13,30]$. We also plan to investigate which scenarios (e.g. a master and a slave) and which interactions would support collaborative interaction in a single arena. Our software implementation can already support several users in the same arena - each user being considered as an obstacle, but collaborative interactions raise multiple challenges [28].

\section{ACKNOWLEDGEMENTS}

We would like to thank M. Teyssier, C. Rigaud, B. Geslain, S. Sakr, M. Serrano, J. Müller, J. Gugenheimer as well as the participants of the studies. 


\section{REFERENCES}

[1] 2019a. CyberGrasp. (2019). http://www. cyberglovesystems . com/cybergrasp

[2] 2019b. Oculus Rift S. (2019). https://www . oculus. com/rift-s/?locale=fr_FR

[3] 2019c. SteamVR - Valve Corporation. (2019). https://www. steamvr.com/en/

[4] 2019d. Teslasuit I Full body haptic VR suit for motion capture and training. (2019). https://teslasuit.io/

[5] Parastoo Abtahi, Benoit Landry, Jackie (Junrui) Yang, Marco Pavone, Sean Follmer, and James A. Landay. 2019. Beyond The Force: Using Quadcopters to Appropriate Objects and the Environment for Haptics in Virtual Reality. In Proceedings of the 2019 CHI Conference on Human Factors in Computing Systems CHI '19. ACM Press, Glasgow, Scotland Uk, 1-13. DOI : http://dx.doi .org/10.1145/3290605. 3300589

[6] E. Amirpour, M. Savabi, A. Saboukhi, M. Rahimi Gorii, H. Ghafarirad, R. Fesharakifard, and S. Mehdi Rezaei. 2019. Design and Optimization of a Multi-DOF Hand Exoskeleton for Haptic Applications. In 2019 7th International Conference on Robotics and Mechatronics (ICRoM). 270-275. DOI :

http://dx.doi.org/10.1109/ICRoM48714.2019.9071884 ISSN: 2572-6889.

[7] Bruno Araujo, Ricardo Jota, Varun Perumal, Jia Xian Yao, Karan Singh, and Daniel Wigdor. 2016. Snake Charmer: Physically Enabling Virtual Objects. In Proceedings of the TEI '16: Tenth International Conference on Tangible, Embedded, and Embodied Interaction - TEI' '16. ACM Press, Eindhoven, Netherlands, 218-226. DOI : http://dx.doi .org/10.1145/2839462.2839484

[8] Jonas Auda, Max Pascher, and Stefan Schneegass. 2019. Around the (Virtual) World: Infinite Walking in Virtual Reality Using Electrical Muscle Stimulation. In Proceedings of the 2019 CHI Conference on Human Factors in Computing Systems - CHI '19. ACM Press, Glasgow, Scotland Uk, 1-8. DOI : http://dx.doi.org/10.1145/3290605.3300661

[9] Mahdi Azmandian, Mark Hancock, Hrvoje Benko, Eyal Ofek, and Andrew D. Wilson. 2016a. Haptic Retargeting: Dynamic Repurposing of Passive Haptics for Enhanced Virtual Reality Experiences. In Proceedings of the $2016 \mathrm{CHI}$ Conference on Human Factors in Computing Systems - CHI '16. ACM Press, Santa Clara, California, USA, 1968-1979. DOI: http://dx.doi.org/10.1145/2858036.2858226

[10] Mahdi Azmandian, Mark Hancock, Hrvoje Benko, Eyal Ofek, and Andrew D. Wilson. 2016b. Haptic Retargeting: Dynamic Repurposing of Passive Haptics for Enhanced Virtual Reality Experiences. In Proceedings of the 2016 CHI Conference on Human Factors in Computing Systems - CHI '16. ACM Press, Santa Clara, California, USA, 1968-1979. DOI : http://dx.doi.org/10.1145/2858036.2858226
[11] Hrvoje Benko, Christian Holz, Mike Sinclair, and Eyal Ofek. 2016. NormalTouch and TextureTouch: High-fidelity 3D Haptic Shape Rendering on Handheld Virtual Reality Controllers. In Proceedings of the 29th Annual Symposium on User Interface Software and Technology - UIST '16. ACM Press, Tokyo, Japan, 717-728. DOI :

http://dx.doi .org/10.1145/2984511.2984526

[12] Gordon Binsted, Romeo Chua, Werner Helsen, and Digby Elliott. 2001. Eye-hand coordination in goal-directed aiming. Human Movement Science 20, 4-5 (Nov. 2001), 563-585. DOI :

http://dx. doi . org/10.1016/S0167-9457(01)00068-9

[13] Scott Brave, Hiroshi Ishii, and Andrew Dahley. 1998. Tangible interfaces for remote collaboration and communication. In Proceedings of the 1998 ACM conference on Computer supported cooperative work CSCW '98. ACM Press, Seattle, Washington, United States, 169-178. DOI :

http://dx. doi .org/10.1145/289444.289491

[14] Steve Bryson. 2005. Direct Manipulation in Virtual Reality. In Visualization Handbook. Elsevier, 413-430. DOI :

http://dx.doi .org/10.1016/B978-012387582-2/50023-X

[15] Lung-Pan Cheng, Li Chang, Sebastian Marwecki, and Patrick Baudisch. 2018. iTurk: Turning Passive Haptics into Active Haptics by Making Users Reconfigure Props in Virtual Reality. In Proceedings of the 2018 CHI Conference on Human Factors in Computing Systems CHI '18. ACM Press, Montreal QC, Canada, 1-10. DOI: http://dx.doi .org/10.1145/3173574.3173663

[16] Lung-Pan Cheng, Patrick Lühne, Pedro Lopes, Christoph Sterz, and Patrick Baudisch. 2014. Haptic Turk: a Motion Platform Based on People. (2014), 11.

[17] Lung-Pan Cheng, Sebastian Marwecki, and Patrick Baudisch. 2017a. Mutual Human Actuation. In Proceedings of the 30th Annual ACM Symposium on User Interface Software and Technology - UIST '17. ACM Press, Qu\&\#233; bec City, QC, Canada, 797-805. DOI: http://dx.doi .org/10.1145/3126594.3126667

[18] Lung-Pan Cheng, Eyal Ofek, Christian Holz, Hrvoje Benko, and Andrew D. Wilson. 2017b. Sparse Haptic Proxy: Touch Feedback in Virtual Environments Using a General Passive Prop. In Proceedings of the 2017 CHI Conference on Human Factors in Computing Systems CHI '17. ACM Press, Denver, Colorado, USA, 3718-3728. DOI : http://dx.doi.org/10.1145/3025453.3025753

[19] Lung-Pan Cheng, Thijs Roumen, Hannes Rantzsch, Sven Köhler, Patrick Schmidt, Robert Kovacs, Johannes Jasper, Jonas Kemper, and Patrick Baudisch. 2015. TurkDeck: Physical Virtual Reality Based on People. In Proceedings of the 28th Annual ACM Symposium on User Interface Software \& Technology - UIST '15. ACM Press, Daegu, Kyungpook, Republic of Korea, 417-426. DOI : http://dx.doi.org/10.1145/2807442.2807463 
[20] Inrak Choi, Heather Culbertson, Mark R. Miller, Alex Olwal, and Sean Follmer. 2017. Grabity: A Wearable Haptic Interface for Simulating Weight and Grasping in Virtual Reality. In Proceedings of the 30th Annual ACM Symposium on User Interface Software and Technology UIST '17. ACM Press, Qu\&\#233;bec City, QC, Canada, 119-130. DOI :

http://dx.doi.org/10.1145/3126594.3126599

[21] Inrak Choi, Eyal Ofek, Hrvoje Benko, Mike Sinclair, and Christian Holz. 2018. CLAW: A Multifunctional Handheld Haptic Controller for Grasping, Touching, and Triggering in Virtual Reality. In Proceedings of the 2018 CHI Conference on Human Factors in Computing Systems - CHI '18. ACM Press, Montreal QC, Canada, 1-13. DOI: http://dx. doi .org/10.1145/3173574.3174228

[22] Xavier de Tinguy, Thomas Howard, Claudio Pacchierotti, Maud Marchal, and Anatole Lécuyer. 2020. WeATaViX: WEarable Actuated TAngibles for VIrtual reality eXperiences. (2020), 9.

[23] Force Dimension. 2019. Force Dimension - products. (2019). http://www. forcedimension. com/

[24] A. Formaglio, A. Giannitrapani, M. Franzini, D. Prattichizzo, and F. Barbagli. 2005. Performance of Mobile Haptic Interfaces. In Proceedings of the 44th IEEE Conference on Decision and Control. 8343-8348. DOI : http://dx.doi .org/10.1109/CDC. 2005.1583513

[25] F. Gosselin, C. Andriot, F. Bergez, and X. Merlhiot. 2007. Widening 6-DOF haptic devices workspace with an additional degree of freedom. In Second Joint EuroHaptics Conference and Symposium on Haptic Interfaces for Virtual Environment and Teleoperator Systems (WHC'07). 452-457. DOI:

http://dx.doi.org/10.1109/WHC.2007.127

[26] Haption. 2019a. Scale1 ${ }^{\text {TM }}$ - HAPTION SA. (2019). https:

//www.haption. com/en/products-en/scale-one-en.html

[27] Haption. 2019b. Virtuose ${ }^{\mathrm{TM}}$ 6D - HAPTION SA. (2019). https:

//www.haption. com/en/products-en/virtuose-6d-en.html

[28] Zhenyi He and Ken Perlin. 2019. CollaboVR: A Reconfigurable Framework for Multi-user to Communicate in Virtual Reality. arXiv:1912.03863 [cs] (Dec. 2019). http://arxiv.org/abs/1912.03863 arXiv: 1912.03863.

[29] Zhenyi He, Fengyuan Zhu, Aaron Gaudette, and Ken Perlin. 2017b. Robotic Haptic Proxies for Collaborative Virtual Reality. arXiv:1701.08879 [cs] (Jan. 2017). http://arxiv.org/abs/1701.08879 arXiv: 1701.08879.

[30] Zhenyi He, Fengyuan Zhu, and Ken Perlin. 2017a. PhyShare: Sharing Physical Interaction in Virtual Reality. arXiv:1708.04139 [cs] (Aug. 2017). http://arxiv.org/abs/1708.04139 arXiv: 1708.04139.

[31] Seongkook Heo, Christina Chung, Geehyuk Lee, and Daniel Wigdor. 2018. Thor's Hammer: An Ungrounded Force Feedback Device Utilizing Propeller-Induced
Propulsive Force. In Proceedings of the 2018 CHI Conference on Human Factors in Computing Systems CHI '18. ACM Press, Montreal QC, Canada, 1-11. DOI : http://dx.doi .org/10.1145/3173574.3174099

[32] Anuruddha Hettiarachchi and Daniel Wigdor. 2016. Annexing Reality: Enabling Opportunistic Use of Everyday Objects as Tangible Proxies in Augmented Reality. In Proceedings of the 2016 CHI Conference on Human Factors in Computing Systems - CHI '16. ACM Press, Santa Clara, California, USA, 1957-1967. DOI : http://dx.doi.org/10.1145/2858036.2858134

[33] Matthias Hoppe, Pascal Knierim, Thomas Kosch, Markus Funk, Lauren Futami, Stefan Schneegass, Niels Henze, Albrecht Schmidt, and Tonja Machulla. 2018. VRHapticDrones: Providing Haptics in Virtual Reality through Quadcopters. In Proceedings of the 17th International Conference on Mobile and Ubiquitous Multimedia - MUM 2018. ACM Press, Cairo, Egypt, 7-18. DOI : http://dx .doi .org/10.1145/3282894.3282898

[34] Matthias Hoppe, Beat Rossmy, Daniel Peter Neumann, Stephan Streuber, Albrecht Schmidt, and Tonja-Katrin Machulla. 2020. A Human Touch: Social Touch Increases the Perceived Human-likeness of Agents in Virtual Reality. (2020), 11.

[35] Hiroshi Hoshino. 1995. A Contruction MEthod of Virtual Haptic Space. (1995). http://www. files.tachilab. org/publications/intconf1900/hoshino199511ICAT.pdf

[36] Hsin-Yu Huang, Chih-Wei Ning, Po-Yao (Cosmos) Wang, Jen-Hao Cheng, and Lung-Pan Cheng. 2020. Haptic-go-round: A surrounding Platform for EncounterType Haptic in VR experienes. (2020). https://dl.acm.org/doi/pdf/10.1145/3334480.3383136

[37] Brent Edward Insko. 2001. Passive Haptics Significantly Enhances Virtual Environments. (2001), 111.

[38] Yaesol Kim, Hyun Jung Kim, and Young J. Kim. 2018. Encountered-type haptic display for large VR environment using per-plane reachability maps: Encountered-type Haptic Display for Large VR Environment. Computer Animation and Virtual Worlds 29, 3-4 (May 2018), e1814. DOI : http://dx.doi.org/10.1002/cav. 1814

[39] Pascal Knierim, Thomas Kosch, Valentin Schwind, Markus Funk, Francisco Kiss, Stefan Schneegass, and Niels Henze. 2017. Tactile Drones - Providing Immersive Tactile Feedback in Virtual Reality through Quadcopters. In Proceedings of the 2017 CHI Conference Extended Abstracts on Human Factors in Computing Systems - CHI EA '17. ACM Press, Denver, Colorado, USA, 433-436. DOI :

http://dx.doi.org/10.1145/3027063.3050426

[40] Chaehyun Lee, Min Sik Hong, In Lee, Oh Kyu Choi, Kyung-Lyong Han, Yoo Yeon Kim, Seungmoon Choi, and Jin S Lee. 2007. Mobile Haptic Interface for Large Immersive Virtual Environments: PoMHI v0.5. (2007), 2. 
[41] In Lee, Inwook Hwang, Kyung-Lyoung Han, Oh Kyu Choi, Seungmoon Choi, and Jin S. Lee. 2009. System improvements in Mobile Haptic Interface. In World Haptics 2009 - Third Joint EuroHaptics conference and Symposium on Haptic Interfaces for Virtual Environment and Teleoperator Systems. IEEE, Salt Lake City, UT, USA, 109-114. DOI :

http://dx.doi .org/10.1109/WHC. 2009.4810834

[42] Jaeyeon Lee, Mike Sinclair, Mar Gonzalez-Franco, Eyal Ofek, and Christian Holz. 2019. TORC: A Virtual Reality Controller for In-Hand High-Dexterity Finger Interaction. In Proceedings of the 2019 CHI Conference on Human Factors in Computing Systems - CHI ' 19. ACM Press, Glasgow, Scotland Uk, 1-13. DOI : http://dx.doi.org/10.1145/3290605.3300301

[43] Jean-Claude Lepecq, Lionel Bringoux, Jean-Marie Pergandi, Thelma Coyle, and Daniel Mestre. 2008. Afforded Actions as a Behavioral Assessment of Physical Presence. (2008), 8.

[44] Pedro Lopes, Alexandra Ion, and Patrick Baudisch. 2015. Impacto: Simulating Physical Impact by Combining Tactile Stimulation with Electrical Muscle Stimulation. In Proceedings of the 28th Annual ACM Symposium on User Interface Software \& Technology - UIST'15. ACM Press, Daegu, Kyungpook, Republic of Korea, 11-19. DOI : http://dx.doi .org/10.1145/2807442.2807443

[45] W. A. McNeely. 1993. Robotic graphics: a new approach to force feedback for virtual reality. In Proceedings of IEEE Virtual Reality Annual International Symposium. 336-341. DOI: http://dx.doi.org/10.1109/VRAIS. 1993.380761

[46] Víctor Mercado and Univ Rennes. 2020. Design and Evaluation of Interaction Techniques Dedicated to Integrate Encountered-Type Haptic Displays in Virtual Environments. (2020), 9.

[47] Kouta Minamizawa, Domenico Prattichizzo, and Susumu Tachi. 2010. Simplified design of haptic display by extending one-point kinesthetic feedback to multipoint tactile feedback. In 2010 IEEE Haptics Symposium. IEEE, Waltham, MA, USA, 257-260. DOI : http://dx.doi.org/10.1109/HAPTIC.2010.5444646

[48] Norbert Nitzsche, Uwe D. Hanebeck, and G. Schmidt. 2003. Design issues of mobile haptic interfaces. Journal of Robotic Systems 20, 9 (Sept. 2003), 549-556. DOI : http://dx.doi.org/10.1002/rob. 10105

[49] J. Pair, U. Neumann, D. Piepol, and B. Swartout. 2003. FlatWorld: combining Hollywood set-design techniques with VR. IEEE Computer Graphics and Applications 23, 1 (Jan. 2003), 12-15. DOI :

http://dx.doi.org/10.1109/MCG. 2003.1159607

[50] Sharif Razzaque, Zachariah Kohn, and Mary C. Whitton. 2001. EUROGRAPHICS 2001 / Jonathan C. Roberts Short Presentation (C) The Eurographics Association 2001. Redirected Walking.

[51] Katja Rogers, Jana Funke, Julian Frommel, Sven Stamm, and Michael Weber. 2019. Exploring Interaction Fidelity in Virtual Reality: Object Manipulation and Whole-Body Movements. In Proceedings of the 2019 CHI Conference on Human Factors in Computing Systems - CHI '19. ACM Press, Glasgow, Scotland Uk, 1-14. DOI : http://dx.doi .org/10.1145/3290605.3300644

[52] Massimo Satler, Carlo A. Avizzano, and Emanuele Ruffaldi. 2011. Control of a desktop mobile haptic interface. In 2011 IEEE World Haptics Conference. IEEE, Istanbul, 415-420. DOI : http://dx.doi.org/10.1109/WHC.2011.5945522

[53] Hasti Seifi, Farimah Fazlollahi, Michael Oppermann, John Andrew Sastrillo, Jessica Ip, Ashutosh Agrawal, Gunhyuk Park, Katherine J. Kuchenbecker, and Karon E. MacLean. 2019. Haptipedia: Accelerating Haptic Device Discovery to Support Interaction \& Engineering Design. In Proceedings of the 2019 CHI Conference on Human Factors in Computing Systems - CHI '19. ACM Press, Glasgow, Scotland Uk, 1-12. DOI : http://dx.doi.org/10.1145/3290605.3300788

[54] Emily Shaw, Tessa Roper, Tommy Nilsson, Glyn Lawson, Sue V. G. Cobb, and Daniel Miller. 2019. The Heat is On: Exploring User Behaviour in a Multisensory Virtual Environment for Fire Evacuation. Proceedings of the 2019 CHI Conference on Human Factors in Computing Systems - CHI '19 (2019), 1-13. DOI : http://dx.doi.org/10.1145/3290605.3300856 arXiv: 1902.04573.

[55] Ken Shigeta, Yuji Sato, and Yasuyoshi Yokokohji. 2007. Motion Planning of Encountered-type Haptic Device for Multiple Fingertips Based on Minimum Distance Point Information. In Second Joint EuroHaptics Conference and Symposium on Haptic Interfaces for Virtual Environment and Teleoperator Systems (WHC'07). IEEE, Tsukaba, 188-193. DOI : http://dx.doi.org/10.1109/WHC.2007.85

[56] Alexa F. Siu, Eric J. Gonzalez, Shenli Yuan, Jason B. Ginsberg, and Sean Follmer. 2018. shapeShift: 2D Spatial Manipulation and Self-Actuation of Tabletop Shape Displays for Tangible and Haptic Interaction. In Proceedings of the 2018 CHI Conference on Human Factors in Computing Systems - CHI '18. ACM Press, Montreal QC, Canada, 1-13. DOI:

http://dx.doi.org/10.1145/3173574.3173865

[57] Evan Strasnick, Christian Holz, Eyal Ofek, Mike Sinclair, and Hrvoje Benko. 2018. Haptic Links: Bimanual Haptics for Virtual Reality Using Variable Stiffness Actuation. In Proceedings of the 2018 CHI Conference on Human Factors in Computing Systems CHI '18. ACM Press, Montreal QC, Canada, 1-12. DOI : http://dx.doi .org/10.1145/3173574.3174218

[58] Ryo Suzuki, Hooman Hedayati, Clement Zheng, James Bohn, Daniel Szafir, Ellen Yi-Luen Do, Mark D Gross, and Daniel Leithinger. 2020a. RoomShift: Room-scale Dynamic Haptics for VR with Furniture-moving Swarm Robots. (2020), 11. 
[59] Ryo Suzuki, Ryosuke Nakayama, Dan Liu, Yasuaki Kakehi, Mark D. Gross, and Daniel Leithinger. 2020b. LiftTiles: Constructive Building Blocks for Prototyping Room-scale Shape-changing Interfaces. In Proceedings of the Fourteenth International Conference on Tangible, Embedded, and Embodied Interaction. ACM, Sydney

NSW Australia, 143-151. DOI :

http://dx.doi.org/10.1145/3374920.3374941

[60] Shan-Yuan Teng, Da-Yuan Huang, Chi Wang, Jun Gong, Teddy Seyed, Xing-Dong Yang, and Bing-Yu Chen. 2019. Aarnio: Passive Kinesthetic Force Output for Foreground Interactions on an Interactive Chair. In Proceedings of the 2019 CHI Conference on Human Factors in Computing Systems - CHI '19. ACM Press, Glasgow, Scotland Uk, 1-13. DOI :

http://dx.doi.org/10.1145/3290605.3300902

[61] Shan-Yuan Teng, Tzu-Sheng Kuo, Chi Wang, Chi-huan Chiang, Da-Yuan Huang, Liwei Chan, and Bing-Yu Chen. 2018. PuPoP: Pop-up Prop on Palm for Virtual Reality. In The 31 st Annual ACM Symposium on User Interface Software and Technology - UIST '18. ACM Press, Berlin, Germany, 5-17. DOI :

http://dx.doi .org/10.1145/3242587.3242628

[62] Shan-Yuan Teng, Cheng-Lung Lin, Chi-huan Chiang, Tzu-Sheng Kuo, Liwei Chan, Da-Yuan Huang, and Bing-Yu Chen. 2019. TilePoP: Tile-type Pop-up Prop for Virtual Reality. (2019), 11.

[63] Marc Teyssier. 2019. Uduino I Home. (2019). https://marcteyssier. com/uduino/

[64] Martin Usoh, Kevin Arthur, Mary C. Whitton, Rui Bastos, Anthony Steed, Mel Slater, and Frederick P. Brooks. 1999. Walking $>$ walking-in-place $>$ flying, in virtual environments. In Proceedings of the 26th annual conference on Computer graphics and interactive techniques - SIGGRAPH '99. ACM Press, Not Known, 359-364. DOI :

http://dx.doi.org/10.1145/311535.311589

[65] Emanuel Vonach, Clemens Gatterer, and Hannes Kaufmann. 2017. VRRobot: Robot actuated props in an infinite virtual environment. In 2017 IEEE Virtual Reality (VR). IEEE, Los Angeles, CA, USA, 74-83. DOI: http://dx.doi .org/10.1109/VR. 2017.7892233

[66] Yuntao Wang, Hanchuan Li, Zhengyi Cao, Huiyi Luo, $\mathrm{Ke} \mathrm{Ou}$, John Raiti, Chun Yu, Shwetak Patel, and Yuanchun Shi. 2020. MoveVR: Enabling Multiform Force Feedback in Virtual Reality using Household Cleaning Robot. (2020), 12.
[67] Alan Wexelblat. 1993. Virtual reality: applications and explorations. (1993). http://libertar.io/lab/ wp-content/uploads/2016/02/Virtual.Reality.- . Applications. And_. Explorations.pdf/page $=164$ Myron Krueger, Artificial reality 2 An easy entry to Virtual reality Chap 7.

[68] Eric Whitmire, Hrvoje Benko, Christian Holz, Eyal Ofek, and Mike Sinclair. 2018. Haptic Revolver: Touch, Shear, Texture, and Shape Rendering on a Reconfigurable Virtual Reality Controller. In Proceedings of the 2018 CHI Conference on Human Factors in Computing Systems - CHI'18. ACM Press, Montreal QC, Canada, 1-12. DOI : http://dx.doi.org/10.1145/3173574.3173660

[69] M. Yafune and Y. Yokokohji. 2011. Haptically rendering different switches arranged on a virtual control panel by using an encountered-type haptic device. In 2011 IEEE World Haptics Conference. 551-556. DOI : http://dx.doi.org/10.1109/WHC. 2011.5945545

[70] Kotaro Yamaguchi, Ginga Kato, Yoshihiro Kuroda, Kiyoshi Kiyokawa, and Haruo Takemura. 2016. A Non-grounded and Encountered-type Haptic Display Using a Drone. In Proceedings of the 2016 Symposium on Spatial User Interaction - SUI '16. ACM Press, Tokyo, Japan, 43-46. DOI : http://dx.doi .org/10.1145/2983310.2985746

[71] Yuan-Syun Ye, Hsin-Yu Chen, and Liwei Chan. 2019. Pull-Ups: Enhancing Suspension Activities in Virtual Reality with Body-Scale Kinesthetic Force Feedback. In Proceedings of the 32nd Annual ACM Symposium on User Interface Software and Technology - UIST '19. ACM Press, New Orleans, LA, USA, 791-801. DOI: http://dx.doi.org/10.1145/3332165.3347874

[72] Yasuyoshi Yokokohji, Ralph L. Hollis, and Takeo Kanade. 1999. WYSIWYF Display: A Visual/Haptic Interface to Virtual Environment. Presence: Teleoperators and Virtual Environments 8, 4 (Aug. 1999), 412-434. DOI :

http://dx.doi .org/10.1162/105474699566314

[73] Y. Yokokohji, J. Kinoshita, and T. Yoshikawa. 2001. Path planning for encountered-type haptic devices that render multiple objects in 3D space. In Proceedings IEEE Virtual Reality 2001. 271-278. DOI: http://dx.doi. org/10.1109/vR.2001.913796

[74] Daniel Zielasko and Bernhard E Riecke. 2020. Either Give Me a Reason to Stand or an Opportunity to Sit in VR. (2020), 3. 